\title{
Statistical continuum theory for large plastic deformation of polycrystalline materials
}

\author{
Brent L. Adams \\ b_I_adams@byu.edu \\ S. Ahzi \\ H. Garmestani
}

S. Lin

Follow this and additional works at: https://scholarsarchive.byu.edu/facpub

Part of the Mechanical Engineering Commons

\section{Original Publication Citation}

Journal of the Mechanics and Physics of Solids 49 (21) 589-67

\section{BYU ScholarsArchive Citation}

Adams, Brent L.; Ahzi, S.; Garmestani, H.; and Lin, S., "Statistical continuum theory for large plastic deformation of polycrystalline materials" (2000). Faculty Publications. 597.

https://scholarsarchive.byu.edu/facpub/597 


\title{
Statistical continuum theory for large plastic deformation of polycrystalline materials
}

\author{
H. Garmestani ${ }^{a, *}$, S. Lin ${ }^{\mathrm{a}}$, B.L. Adams ${ }^{\mathrm{b}}$, S. Ahzi ${ }^{\mathrm{c}}$ \\ ${ }^{a}$ Department of Mechanical Engineering, Mechanical Engineering at the FAMU-FSU \\ College of Engineering, Center for Materials Research and Technology (MARTECH), \\ Tallahassee, FL 32310, USA \\ ${ }^{\mathrm{b}}$ Brigham Young University, Provo, UT 84602, USA \\ ${ }^{\mathrm{c}}$ Universite Louis Pasteur, Strasbourg, France
}

\begin{abstract}
This paper focuses on the application of statistical continuum mechanics to the prediction of mechanical response of polycrystalline materials and microstructure evolution under large plastic deformations. A statistical continuum mechanics formulation is developed by applying a Green's function solution to the equations of stress equilibrium in an infinite domain. The distribution and morphology of grains (crystals) in polycrystalline materials is represented by a set of correlation functions that are described by the corresponding probability functions. The elastic deformation is neglected and a viscoplastic power law is employed for crystallographic slip in single crystals. In this formulation, two- and three-point probability functions are used. A secant modulus-based formulation is used. The statistical analysis is applied to simulate homogeneous deformation processes under uniaxial tension, uniaxial compression and plane strain compression of an FCC polycrystal. The results are compared to the well-known Taylor upper bound model and discussed in comparison to experimental observations.
\end{abstract}

Keywords: A. Microstructures; B. Polycrystalline; C. Probability and statistics

\section{Introduction}

The formulation of the macroscopic mechanical response of polycrystalline medium has been the subject of many studies and approaches (Ahzi, 1987; Budiansky, 1965; Hashin and Shtrikman, 1962; Hutchinson, 1976; Kröner, 1972; Morris, 1970; Reuss, 1929; Voigt, 1889). Applying statistical continuum theory in predicting elastic

\footnotetext{
${ }^{*}$ Corresponding author. Tel.: +1-850-487-6167; fax: +1-850-644-9281.

E-mail address: garm@magnet.fsu.edu (H. Garmestani).
} 
properties of polycrystals was initiated independently by Beran and Molyneux, Volkov and Klinskikh (1965) and Klinskikh (1965), Lomakin (1970), and Kröner (1967). In this theory, the elastic moduli of polycrystals are estimated from the correlation functions (Beran and Molyneux, 1968). The correlation functions are the means through which the statistical concepts are incorporated into the model. In contrast to the selfconsistent theory (Hill, 1965; Molinari et al., 1987), the statistical formulation is explicit, which means that the theory provides the complete macroscopic constitutive law, linking the applied stress and the macroscopic strain-rate tensors in closed form, if the correlation functions are provided.

A comprehensive work on the statistical theory has been developed primarily by Beran (Beran and Molyneux, 1968; Beran and Molyneux, 1966; Lomakin, 1970; Kröner, 1972). Their contribution produced a basis to apply the theory to heterogeneous materials. Kröner $(1967,1987)$ improved the statistical theory with attention to mathematical aspects of the theory of elasticity. The application to anisotropic materials was studied by Zeller and Dederichs (1973). McCoy (1981) focused on the relationship between the substructure geometry and the correlation functions.

Due to the great practical interest in the large deformation polycrystalline theory, a lot of effort has been devoted to develop appropriate approaches. In general, at large deformations, the elastic strain is neglected. As early as 1938, Taylor proposed a uniform strain model that assumes the plastic strain in each grain is identical to the macroscopic plastic strain (Taylor, 1938). Models based upon Taylor's assumption (Asaro and Needleman, 1985; Bishop and Hill, 1951; Hutchinson, 1976; Kocks and Canova, 1981) have often demonstrated first-order agreement with the measurement of mechanical anisotropy of polycrystalline materials. However, the hypothesis of plastic strain uniformity is somewhat crude; it has been shown to fail when plastic strain heterogeneities are evident. One the so-called "relaxed constraints" theory (Honneff and Mecking, 1978; Houtte, 1981; Kocks and Canova, 1981; Leffers, 1968) takes into account the strain heterogeneities produced by anisotropic grain shapes and the predicted results have a better agreement with experiments. This is true particularly in some specific cases, such as large strain rolling and torsion of fcc metals. Asaro and Needleman (1985) proposed an extension of Taylor theory for large deformations including elastic deformations. It should be noticed that all theories based on Taylor's strain uniformity only fulfill compatibility but not equilibrium conditions at grain boundaries.

In 1987, Molinari et al. (1987) proposed a self-consistent approach for the large deformation polycrystal viscoplasticity. Their approach was based on a scheme developed by Zeller and Dederichs (1973) in heterogeneous elasticity. In this approach, equilibrium and compressibility equations are used to arrive at an integral equation for the local velocity gradient. This integral equation can be solved via different approximate schemes. In the self-consistent model of Molinari et al. (1987), a single crystal (grain) is considered as an inclusion embedded in a homogeneous equivalent medium. The interaction law derived from the integral equation results in a nonlinear relation between stress and strain rate that is solved by a straightforward Newton method. We note that Nemat-Nasser and Obata (1986) also proposed a self-consistent model for large elastic-viscoplastic polycrystalline deformation by using Hill's self-consistent scheme 
(Hill, 1965). However, this model was shown to predict similar results to those of the Taylor upper bound at large deformations (Harren, 1991).

The statistical continuum theory also plays an equally important role with selfconsistent model in the plasticity of large deformation as it did in elasticity. Adams et al. (1989) presented a statistical formulation of viscoplastic behavior in heterogeneous polycrystals by taking the approach which parallels the constructs in the statistical continuum theory of linear-elastic polycrystals (Kröner, 1967, 1972, 1987; McCoy, 1981). A secant modulus formulation of the single-crystal constitutive law was used. The interaction law from equilibrium conditions and the incompressibility condition was obtained by using Green's function method. The statistical formulation was formed from the interaction law by incorporating the 2-point probability density function of lattice orientations, that can also be called the crystallite orientation correlation function (OCF) (Adams et al., 1988). The statistical theory of Adams et al. (1989) was first applied to the prediction of initial texture evolution in FCC polycrystals under uniaxial creep (Adams and Field, 1991). Evolution of the correlation functions was not considered in this initial work.

The traditional analysis of polycrystalline orientation uses the crystallite orientation distribution function (ODF), which is an average representation of polycrystalline lattice orientation, to define the texture. Existing polycrystalline plasticity models account for the initial ODF, and predict its evolution, but completely neglect the spatial correlation between crystals. The statistical modeling proposed in this paper, based on the work of Adams et al. (1989), accounts for not only lattice orientations but also their spatial correlations. For the formulation of the correlation between crystals, a 2-point probability density function, named the orientation coherence function (OCF), is advocated as a logical first step towards more sophisticated microstructural measures (Adams and Field, 1991; Adams et al., 1987, 1988; Morris et al., 1988). The OCF describes the correlation of lattice orientations between points in the polycrystalline material separated by a specified vector. The OCF requires nine parameters - three describing the lattice orientation at each point, and three defining their spatial relationship with respect to one another, whereas the ODF can be conveniently defined on a three-dimensional manifold parameterized by Euler angles for each crystal. Measurements of the OCF are much more demanding than those required for the ODF. However, with simultaneous advances in micro-diffraction technology and computing hardware and software, OCF measurement is now possible for many crystalline materials (Adams et al., 1993; Wright et al., 1993).

It could be stated that the main problem in the implementation of the statistical model for large deformation plasticity of polycrystalline materials is the incorporation of the OCF in the analyses. This problem consists of two parts: OCF measurement, and closed form representation of the OCF for certain types of material configurations. During the 1990s, a new technique, called orientation imaging microscopy (OIM), was developed for examining the spatial arrangement of lattice orientations in polycrystalline microstructures. The orientation of the crystal lattice can be determined from electron backscatter diffraction patterns (EBSPs) in the SEM. OIM was first described by Adams et al. (1993) and the initial applications of OIM to polycrystalline aluminum was described by Wright et al. (1993). OIM data can be directly interrogated to obtain 
estimates of the OCF. These estimates procure naturally in tabular form, expressing the probabilities as statistical fractions dependent upon nine degrees of freedom. If incorporated directly into the numerical calculation, the relevant procedures involve an enormous computational time. Closed-form representations for the OCF are preferred in the numerical procedure. The parameters contained in the formulation can be derived from the experimental data by an appropriate numerical fitting method.

Corson $(1976 \mathrm{a}, \mathrm{b})$ proposed a form for the representation of the two- and three-point probability functions for a two-isotropic-phase medium. Irrespective of the simplicity of the case dealt with by corson, the application of this form allows a better understanding of the problem and the derivation of more complicated forms. This form of the probability function had been applied to estimate the inelastic effective properties of a two-phase medium (Garmestani et al., 1998). In a later work, the evolution of two-point probability functions has been investigated for composite materials (Lin et al., 2000). The effective properties of an elastic two-phase medium was investigated separately by Garmestani and Lin (2000). For a polycrystalline microstructure, the proper functional form of the probability function cannot be derived as easily since nine variables have to be included for a two-point OCF. The work presented here to describe the two-point $\mathrm{OCF}$, for a heterogeneous polycrystalline medium, is essentially an extension of Corson's work. In the statistical continuum model for polycrystals, the calculation of texture evolution involves three-point probability functions. However, in this paper an approximation for the decomposition of the three-point probability functions using the two-point probability functions is introduced.

This paper focuses on the application of statistical continuum mechanics to large deformation plasticity of polycrystalline materials. Elasticity is neglected and plasticity is assumed to occur by crystallographic slip. A viscoplastic power law is assumed for crystallographic slip and used to derive the three-dimensional constitutive relation for single-crystal viscoplasticity. In Section 2, we briefly review the single-crystal viscoplasticity and use the secant modulus formulation for the description of the single-crystal behavior. In Section 3, we show how the local velocity gradient is linked to the macroscopic one (interaction law) using the integral method which follows the scheme developed by Molinari et al. (1987). Section 4 shows the description of the proposed first-order statistical concept, which leads to the derivation of the statistical interaction law, and the description of the two-point correlation functions. In Section 4, the evolution of texture and the two-point correlation functions are described via simplified three-point probability functions that are approximated from the two-point correlation functions. In the last section, simulated results for an FCC metal under homogeneous deformation are shown for uniaxial and plane strain loading conditions. These results are discussed in comparison to experimentally observed ones and are directly compared to the results from the Taylor upper-bound model.

\section{Interaction law}

In the following, the interaction law is derived for a viscoplastic polycrystal using the integral equation as formulated by Molinari et al. (1987). Note that elasticity is 
neglected and the secant formulation is used. We denote by $\mathbf{T}$ the local Cauchy stress, $\mathbf{T}=\mathbf{S}-p \mathbf{I}$, with $p$ representing the pressure and $\mathbf{I}$ is the second-order identity tensor. Because of the full symmetry of $\mathbf{M}$, the secant modulus $\mathbf{N}$ is also fully symmetric. If $\mathbf{L}$ represents the velocity gradient, with $\mathbf{D}$ the symmetric part of $\mathbf{L}$, Eq. (7) can be written as $\mathbf{S}=\mathbf{N} \mathbf{L}$. Therefore, the equilibrium equation throughout the polycrystal can be expressed as

$$
T_{i j, j}=\left(N_{i j k l} L_{k l}\right)_{, j}-p_{, i}=0 .
$$

Locally $\mathbf{N}$ is determined by $\mathbf{L}$ and a set of state variables, $h$. Here, $h$ includes the three Euler angles, which define the orientation of the crystal lattice with respect to a chosen macroscopic reference frame. Decompose $\mathbf{N}$ as the sum of a uniform part $\mathbf{N}^{0}$ and a space-dependent part $\tilde{\mathbf{N}}$ to obtain $\mathbf{N}=\mathbf{N}^{0}+\tilde{\mathbf{N}}$. The uniform fourth-order tensor $\mathbf{N}^{0}$ is assumed to represent the instantaneous secant moduli of the homogeneous equivalent medium (HEM). Molinari et al. (1987) proposed a Green's function solution for the set of PDEs represented by Eq. (1) and the compatibility conditions, $L_{i i}=0$ :

$$
\begin{aligned}
L_{i k}(\mathbf{r}) & =\bar{L}_{i k}+\int_{r^{\prime} \in V} G_{i j, k}\left(\mathbf{r}-\mathbf{r}^{\prime}\right)\left[\tilde{N}_{j l r s}\left(L\left(\mathbf{r}^{\prime}\right), h\left(\mathbf{r}^{\prime}\right)\right) L_{r s}\left(\mathbf{r}^{\prime}\right)\right]_{, l} \mathrm{~d} \boldsymbol{r}^{\prime} \\
& =\bar{L}_{i k}+\int_{r^{\prime} \in V} G_{i j, k l}\left(\mathbf{r}-\mathbf{r}^{\prime}\right) \tilde{N}_{j l r s}\left(L\left(\mathbf{r}^{\prime}\right), h\left(\mathbf{r}^{\prime}\right)\right) L_{r s}\left(\mathbf{r}^{\prime}\right) \mathrm{d} \boldsymbol{r}^{\prime},
\end{aligned}
$$

where $G_{i j, k l}\left(\mathbf{r}-\mathbf{r}^{\prime}\right)$ has a singular value at $\mathbf{r}=\mathbf{r}^{\prime}$. A proper solution to this problem is to construct solutions for a finite small volume $V_{\mathrm{c}}$ surrounding point $\mathbf{r}$, then calculate the average value of the velocity gradient to replace the value at $\mathbf{r}$ (Kröner, 1987). Hence, a very small volume (compared to the volume of the whole specimen) is used in order to obtain a solution (Garmestani et al., 1998; Lin et al., 2000). Let $\mathbf{L}^{0}(\mathbf{r})$ be this averaged value

$$
L_{i k}^{0}(\mathbf{r})=\bar{L}_{i k}+\frac{1}{V_{\mathrm{c}}} \int_{r \in V_{\mathrm{c}}} \int_{r^{\prime} \in V} G_{i j, k l}\left(\mathbf{r}-\mathbf{r}^{\prime}\right) \tilde{N}_{j l r s}\left(\mathbf{L}^{0}\left(\mathbf{r}^{\prime}\right), h\left(\mathbf{r}^{\prime}\right)\right) L_{r s}^{0}\left(\mathbf{r}^{\prime}\right) \mathrm{d} \boldsymbol{r}^{\prime} \mathrm{d} \boldsymbol{r} .
$$

Using the abbreviated form of Eq. (3):

$$
\mathbf{L}^{0}(\mathbf{r})=\overline{\mathbf{L}}+\mathbf{G}\left(\mathbf{r}-\mathbf{r}^{\prime}\right) * \tilde{\mathbf{N}}\left(\mathbf{r}^{\prime}\right) \mathbf{L}^{0}\left(\mathbf{r}^{\prime}\right) .
$$

The symmetric part and the antisymmetrical part of Eq. (4) define the local strain-rate tensor $\mathbf{D}^{0}(\mathbf{r})$ and the local total spin tensor $\mathbf{W}^{0}(\mathbf{r})$, respectively. The total spin tensor consists of a lattice spin and a plastic spin. The rate of lattice rotation $\mathbf{W}^{0 *}(\mathbf{r})$ is the lattice spin and the plastic part $\mathbf{W}^{0 \mathrm{p}}(\mathbf{r})$ does not change the orientation of crystal lattice, thus

$$
\mathbf{W}^{0 *}(\mathbf{r})=\mathbf{W}^{0}(\mathbf{r})-\mathbf{W}^{0 \mathrm{p}}(\mathbf{r}),
$$

where $W_{i j}^{0 \mathrm{p}}=\frac{1}{2} \sum_{\alpha}\left(s_{i}^{\alpha} n_{j}^{\alpha}-s_{j}^{\alpha} n_{i}^{\alpha}\right) \dot{\gamma}^{\alpha}$. The unit vectors $\mathbf{s}^{\alpha}$ and $\mathbf{n}^{\alpha}$ represent, respectively, the slip direction and the slip-plane normal for the $\alpha$ th slip system. The slip rate for the $\alpha$ th slip system, $\dot{\gamma}^{\alpha}$, can be calculated using (Hutchinson, 1976)

$$
\tau^{\alpha} / \tau_{\mathrm{c}}^{\alpha}=\left(\dot{\gamma}^{\alpha} / \dot{\gamma}_{0}\right)^{m}
$$


where $m$ is the rate sensitivity parameter, and $\tau_{\mathrm{c}}^{\alpha}$ and $\dot{\gamma}_{0}$ represent the critical shear stress and a reference shear strain rate, respectively. The strain rate in the single crystal, $\mathbf{D}$, is equal to the plastic strain rate, which is given by the sum over the contributions from all slip systems. It is related to the deviatoric Cauchy stress tensor $\mathbf{S}$ by

$$
\mathbf{D}_{i j}=\mathbf{M}_{i j k l} \mathbf{S}_{k l}
$$

where

$$
M_{i j k l}=\sum_{\alpha}\left(\dot{\gamma}_{0} / \tau_{\mathrm{c}}^{\alpha}\right)\left(\mu_{\mathrm{rs}}^{\alpha} S_{\mathrm{rs}} / \tau_{\mathrm{c}}^{\alpha}\right)^{n-1} \mu_{i j}^{\alpha} \mu_{k l}^{\alpha}
$$

and

$$
\mu_{i j}^{\alpha}=\frac{1}{2}\left(s_{i}^{\alpha} n_{j}^{\alpha}+s_{j}^{\alpha} n_{i}^{\alpha}\right)
$$

and $n=1 / m$. The fourth-order compliance tensor $\mathbf{M}$ is positive definite and the deviatoric stress and deviatoric strain-rate relation (5) is a one-to-one relation. Therefore, the inverse relationship to this equation exists where $\mathbf{N}=\mathbf{M}^{-1}$.

\section{Statistical formulation and correlation functions}

Assume that velocity gradient field does not deviate too far from the uniform field of the Taylor-type polycrystal. Using Taylor series expansion about $\overline{\mathbf{L}}$, Eq. (4) can be written as

$$
L^{0}=\bar{L}+G *\left(\tilde{\sigma}(\bar{L})+\tilde{\sigma}^{\prime}(\bar{L}) \tilde{L}^{0}+\frac{1}{2 !} \tilde{\sigma}^{\prime \prime}(\bar{L})\left(\tilde{L}^{0}\right)^{2}+\cdots\right),
$$

where $\tilde{\sigma}$ is the polarized deviatoric stress defined by

$$
\tilde{\sigma}_{j l}\left(\mathbf{r}^{\prime}\right)=\tilde{N}_{j l r s}\left(\mathbf{L}\left(\mathbf{r}^{\prime}\right), h\left(\mathbf{r}^{\prime}\right)\right) L_{r s}\left(\mathbf{r}^{\prime}\right) .
$$

Considering only the first-order correction to the homogeneous Taylor's solution, then Eq. (4) becomes

$$
\mathbf{L}^{0}(\mathbf{r})=\overline{\mathbf{L}}+\mathbf{G}\left(\mathbf{r}-\mathbf{r}^{\prime}\right) * \tilde{\mathbf{N}}\left(\overline{\mathbf{L}}, h\left(\mathbf{r}^{\prime}\right)\right) \overline{\mathbf{L}} .
$$

In the following the statistical concept is introduced with proper correlation functions to relate the local to global properties. Consider the ensemble average of the local velocity gradients for many particles belonging to the same state $h$. Each grain is surrounded by a structure that is different from all other grains even if they are at the same state. This results in a distinctive velocity gradient for each grain. Here we neglect the difference between the local velocity gradient belonging to the grains with the same state and assume that all these grains have one local velocity gradient value that is calculated from the ensemble average. Symbol \langle\rangle$_{h}$ denotes the ensemble average over grains at state $h$. The averages of interest here are obtained from expressions like

$$
\left\langle()_{i}\right\rangle=\frac{1}{N} \sum_{i=1}^{N}()_{i}
$$


Applying this to Eq. (12), the ensemble average of the local velocity gradients belonging to the same state, $h$, can be calculated:

$$
\left\langle\mathbf{L}^{0}(\mathbf{r})\right\rangle_{h}=\overline{\mathbf{L}}+\mathbf{G}\left(\mathbf{r}-\mathbf{r}^{\prime}\right) *\left\langle\tilde{\mathbf{N}}\left(\overline{\mathbf{L}}, h\left(\mathbf{r}^{\prime}\right)\right)\right\rangle_{h} \overline{\mathbf{L}} .
$$

The symmetric part and the antisymmetric part of Eq. (14) correspond to the ensemble average of the local strain rate and local spin tensor, respectively

$$
\begin{aligned}
& \left\langle\mathbf{D}^{0}(\mathbf{r})\right\rangle_{h}=\overline{\mathbf{D}}+\Gamma\left(\mathbf{r}-\mathbf{r}^{\prime}\right) *\left\langle\tilde{\mathbf{N}}\left(\overline{\mathbf{L}}, h\left(\mathbf{r}^{\prime}\right)\right)\right\rangle_{h} \overline{\mathbf{L}}, \\
& \left\langle\mathbf{W}^{0}(\mathbf{r})\right\rangle_{h}=\overline{\mathbf{W}}+\Lambda\left(\mathbf{r}-\mathbf{r}^{\prime}\right) *\left\langle\tilde{\mathbf{N}}\left(\overline{\mathbf{L}}, h\left(\mathbf{r}^{\prime}\right)\right)\right\rangle_{h} \tilde{\mathbf{L}} .
\end{aligned}
$$

Eq. (15) represents the first-order statistical localization law. The correlation function $\left\langle\tilde{\mathbf{N}}\left(\overline{\mathbf{L}}, h\left(\mathbf{r}^{\prime}\right)\right)\right\rangle_{h}$ can be described in terms of the conditional two-point probability density function of state, $h$,

$$
\left\langle\tilde{\mathbf{N}}\left(\overline{\mathbf{L}}, h\left(\mathbf{r}^{\prime}\right)\right)\right\rangle_{h}=\int f\left(\mathbf{r}^{\prime} \in h\left(\mathbf{r}^{\prime}\right) \mid \mathbf{r} \in h\right) \tilde{\mathbf{N}}\left(\overline{\mathbf{L}}, h\left(\mathbf{r}^{\prime}\right)\right) \mathrm{d} h\left(\mathbf{r}^{\prime}\right),
$$

where $f\left(\mathbf{r}^{\prime} \in h\left(\mathbf{r}^{\prime}\right) \mid \mathbf{r} \in h\right)$ is defined as the probability of occurrence of $\mathbf{r}^{\prime}$ at state $h(\mathbf{r})^{\prime}$ given that $\mathbf{r}$ belongs to state $h$.

The polycrystalline material contains a number of grains, each of which can be considered a different phase distinguished by its orientation. Each grain with a different orientation can be considered as belonging to a different state, $h_{i}$. The corresponding two-point probability functions can then be defined according to these states:

$$
P_{i j}= \begin{cases}V_{i}^{2}+V_{i}\left(1-V_{i}\right) \exp \left(-c_{i j} r^{n_{i j}}\right), & i=j, \\ V_{i} V_{j}-V_{i} V_{j} \exp \left(-c_{i j} r^{n_{i j}}\right), & i \neq j,\end{cases}
$$

where, $V_{i}$ and $V_{j}$ represent the volumes fractions of grains belonging to state $h_{i}$ and $h_{j}$, respectively, $c_{i j}$ and $n_{i j}$ are the constants describing the information of grains distribution and morphology for the individual states. Initially, for a microstructure, $c_{i j}$ and $n_{i j}$ are empirical constants determined by a least-squares curve fit between measurement data and the assumed form of the function $P_{i j}$. The conditional two-point correlation function of lattice orientations in Eq. (17) is related to $P_{i j}$ by the following:

$$
f\left(\mathbf{r}^{\prime} \in h_{j} \mid \mathbf{r} \in h_{i}\right)=P_{i j} / V_{i} .
$$

\section{Evolution of the correlation functions}

During deformation, the texture evolves and results in a change in the macroscopic properties (mechanical, etc.) of the polycrystalline material. In the statistical continuum model, the correlation function, which is expressed by the corresponding probability function, is used to describe the microstructure of the polycrystal. The evolution of probability functions with deformation reflects the development of texture. In this section, the factors that influence the evolution of probability functions are evaluated, and a numerical scheme corresponding to their calculation is presented. 


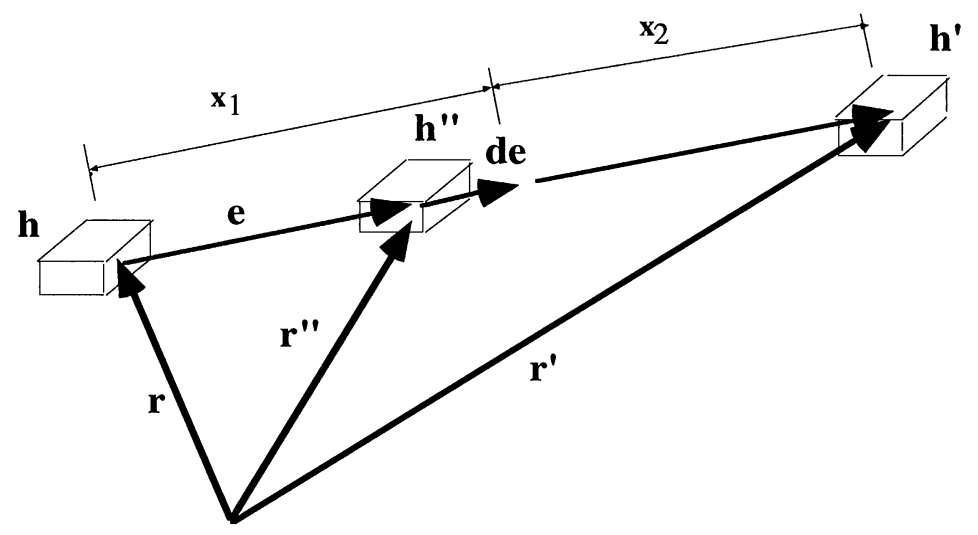

Fig. 1. Definition of parameters associated with the evolution equations.

\subsection{The effect of strain-rate}

The local velocity gradient consists of the strain-rate tensor and the spin tensor. Under an applied strain rate $D_{i j}$ the structure changes and the magnitude of any vector located inside the polycrystal would change accordingly. As a result, parameters $c_{i j}$ and $n_{i j}$ in Eq. (18) are expected to evolve because of this factor.

Referring to Fig. 1, a representative vector $\mathbf{e}$ is shown that originates from state $h$ and terminates at state $h^{\prime}$. The rate of change of the magnitude of e can be written as

$$
\langle\dot{\mathbf{e}}\rangle_{h, h^{\prime}}=\int\left\langle\mathbf{L}\left(\mathbf{r}^{\prime \prime}\right)\right\rangle_{h, h^{\prime}} \mathrm{d} e,
$$

where $\left\langle\mathbf{L}\left(\mathbf{r}^{\prime \prime}\right)\right\rangle_{h, h^{\prime}}$, is the local strain rate represented by vector $\mathbf{r}^{\prime \prime}$ when vector $\mathbf{r}$ belongs to state $h$ and vector $\mathbf{r}^{\prime}$ belongs to state $h^{\prime}$. Consequently, a three-point probability function is needed to obtain the value for $\left\langle\mathbf{L}\left(\mathbf{r}^{\prime \prime}\right)\right\rangle_{h, h^{\prime}}$, from $\left\langle\mathbf{L}\left(\mathbf{r}^{\prime \prime}\right)\right\rangle_{h^{\prime \prime}}$, which is the expected local velocity gradient when the point represented by $\mathbf{r}^{\prime \prime}$ belongs to state $h^{\prime \prime}$ :

$$
\left\langle\mathbf{L}\left(\mathbf{r}^{\prime \prime}\right)\right\rangle_{h, h^{\prime}}=\int f_{3}\left(h^{\prime \prime}, \mathbf{r}^{\prime \prime} \mid h^{\prime}, \mathbf{r}^{\prime}, h, \mathbf{r}\right)\left\langle\mathbf{L}\left(\mathbf{r}^{\prime \prime}\right)\right\rangle_{h^{\prime \prime}} \mathrm{d} h^{\prime \prime} .
$$

Here $f_{3}\left(h^{\prime \prime}, \mathbf{r}^{\prime \prime} \mid h^{\prime}, \mathbf{r}^{\prime}, h, \mathbf{r}\right)$ is a three-point probability function and corresponds to the probability of occurrence of state $h^{\prime \prime}$ at $\mathbf{r}^{\prime \prime}$ given that state $h$ and $h^{\prime}$ occur at $\mathbf{r}$ and $\mathbf{r}^{\prime}$, respectively. This three-point probability function is derived from the two-point probability functions as shown in the following.

Following the earlier work (Adams et al., 1989; Adams and Field, 1991), approximation for the decomposition of the three-point probability function using the two-point probability functions is introduced here as

$$
f_{3}\left(h^{\prime \prime}, \mathbf{r}^{\prime \prime} \mid h^{\prime}, \mathbf{r}^{\prime}, h, \mathbf{r}\right) \cong \frac{x_{1}}{x_{1}+x_{2}} f_{2}\left(h^{\prime \prime}, \mathbf{r}^{\prime \prime} \mid h^{\prime}, \mathbf{r}^{\prime}\right)+\frac{x_{2}}{x_{1}+x_{2}} f_{2}\left(h^{\prime \prime}, \mathbf{r}^{\prime \prime} \mid h, \mathbf{r}\right),
$$

where $x_{1}$ and $x_{2}$ are the magnitudes of the vectors represented by $\left|\mathbf{r}^{\prime \prime}-\mathbf{r}\right|$, and $\left|\mathbf{r}^{\prime \prime}-\mathbf{r}^{\prime}\right|$. Once the rate of change of the magnitude of $\mathbf{e}$ is obtained by Eq. (45), it can be 
integrated with time $t$ as shown by Eq. (48), to provide the change in the magnitude of vector e from the initial point.

$$
\langle\Delta \boldsymbol{e}\rangle_{h, h^{\prime}}=\int_{0}^{t}\langle\dot{\mathbf{e}}\rangle_{h, h^{\prime}} \mathrm{d} t
$$

To determine the constants $c_{i j}$ and $n_{i j}$ in Eq. (18) at each step of the numerical simulation process, $P_{i j}$ 's are assumed to be known on the left-hand side of Eq. (18). The major inputs will be $r$ (the new magnitude of vector $\mathbf{e}$ ) on the right-hand side of Eq. (18). As a result, a simultaneous set of equations in terms of different $r$ inputs are obtained. The new unknown parameters $c_{i j}$ and $n_{i j}$ can now be solved from this set of equations.

\subsection{The effect of spin-tensor}

The grains are divided into groups (states) according to three Euler angles of crystal lattice orientation: $\varphi_{1}, \phi$ and $\varphi_{2}$. Other aspects of the state, such as the critical resolved stress, $\tau_{\mathrm{c}}^{\alpha}$, are held constant in the present work. During deformation, the crystal lattice rotates, and Euler angels change accordingly. The elastic part $\mathbf{W}^{0}(\mathbf{r})$ of the spin tensor $\mathbf{W}^{0}(\mathbf{r})$ reflects the rate of crystal lattice rotation. Each group of grains corresponding to each state provides a particular total spin tensor described by Eq. (16), and therefore has different rate of crystal lattice rotation. The rate of change of crystallographic axes, for instance the a-axis, is given by (Lee et al., 1995)

$$
\mathbf{a}=\mathbf{W}^{0 *} \cdot \mathbf{a} \text {. }
$$

The solution to this equation can be given in incremental form as

$$
\mathbf{a}(t+\Delta t)=\exp \left(\mathbf{W}^{0 *} \Delta t\right) \cdot \mathbf{a}(t),
$$

where $t$ designates time and $\Delta t$ is the time increment. By using the Cayley-Hamilton theorem, the expansion of $\exp (\hat{\mathbf{W}})$, with $\hat{\mathbf{W}}=\mathbf{W}^{0 *} \Delta t$, can be expressed in the following form:

$$
\exp (\hat{\mathbf{W}})=\mathbf{I}+\frac{\sin \omega}{\omega} \hat{\mathbf{W}}+\frac{1-\cos \omega}{\omega^{2}} \hat{\mathbf{W}}^{2},
$$

where $\mathbf{I}$ is the second-order identity tensor and $\omega^{2}=-\frac{1}{2} \operatorname{tr}\left(\hat{\mathbf{W}}^{2}\right)$.

From Eqs. (25) and (26), the changes of Euler angles of grains can be determined. When the three Euler angles of the grains of a specific state change by a set of values, a fraction of these grains will move to a different state according to previous grouping rule. As a result of this process, the volume fractions used in the probability functions in Eq. (18) for corresponding states will change. Again, the probability functions need to be modified according to the changes of all volume fractions.

\section{Simulation and results}

The critical step in solving Eq. (14) is to solve the convolution integration $\mathbf{G}\left(\mathbf{r}-\mathbf{r}^{\prime}\right) *$ $\left\langle\tilde{\mathbf{N}}\left(\overline{\mathbf{L}}, h\left(\mathbf{r}^{\prime}\right)\right)\right\rangle_{h} \overline{\mathbf{L}}$. We choose a cubic element representing a subset of the polycrystalline 


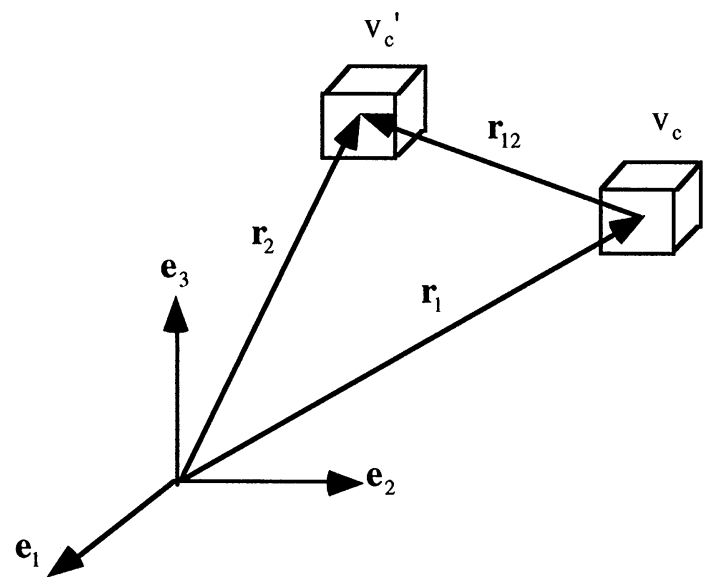

Fig. 2. Two elements geometry of the convolution integral problem $\mathbf{G} * \mathbf{F}$.

medium. Then the cube is divided into $N \times N \times N=N^{3}(N=15$ in the following simulations) of smaller cubes of volume $\Delta^{3}$ shown as $V_{\mathrm{c}} \& V_{\mathrm{c}}^{\prime}$ in Fig. 2. Generally, there are three conditions in this numerical process. First, the cubic region must contain a sufficient number of grains so that it can be taken as a correct statistical representation of the entire specimen. Second, the number of unit cubes is estimated based on a representative grain or size scale within the microstructure such that the average size of such grains are bigger than the size of the unit cube. Third, the cubic region must be large enough so that the corresponding Green's function for the maximum $\left|\mathbf{r}-\mathbf{r}^{\prime}\right|$ falls within the expected error range. For a fixed $\mathbf{r}$ at $V_{\mathrm{c}}$, a second cube of volume $V_{\mathrm{c}}^{\prime}$ that contains $\mathbf{r}^{\prime}$ is considered. The full integral around $\mathbf{r}^{\prime} \in V$ can be taken as the summation of all cubes $V_{\mathrm{c}}^{\prime} \in V$ except $V_{\mathrm{c}}$. Then

$$
\mathbf{G}\left(\mathbf{r}-\mathbf{r}^{\prime}\right) *\left\langle\tilde{\mathbf{N}}\left(\overline{\mathbf{L}}, h\left(\mathbf{r}^{\prime}\right)\right)\right\rangle_{h} \overline{\mathbf{L}}=\sum_{v_{\mathrm{c}}^{\prime} \in V}\left\{\frac{F_{j l}}{V_{\mathrm{c}}} \int_{\mathbf{r} \in V_{\mathrm{c}}} \int_{\mathbf{r}^{\prime} \in V_{\mathrm{c}}^{\prime}} G_{i j, k l}\left(\mathbf{r}-\mathbf{r}^{\prime}\right) \mathrm{d} \boldsymbol{r}^{\prime} \mathrm{d} \boldsymbol{r}\right\},
$$

where $F_{j l}=\left\langle\tilde{\mathbf{N}}\left(\overline{\mathbf{L}}, h\left(\mathbf{r}^{\prime}\right)\right)\right\rangle_{h} \overline{\mathbf{L}}$ is taken to be constant over $V_{\mathrm{c}}^{\prime}$, which means that one cube belongs to one state. $\mathbf{F}$ is related to the probability functions through

$$
\mathbf{F}\left(h_{\mathrm{n}}\right)=\langle\tilde{\mathbf{N}}(\overline{\mathbf{L}}, h)\rangle_{h_{n}} \overline{\mathbf{L}}=\sum_{h} f\left(\mathbf{r}^{\prime} \in h \mid \mathbf{r} \in h_{h}\right) \tilde{\mathbf{N}}(\overline{\mathbf{L}}, h) \overline{\mathbf{L}},
$$

where $f$ is the conditional two-point probability function of lattice orientations and has the relationship shown by Eq. (17) with the two-point probability function $P_{i j}$.

In Eqs. (27) and (28), $h$ and $h_{n}$ are state variables which are determined by orientations of grains. Three Euler angles, $\varphi_{1}, \phi$ and $\varphi_{2}$, in the space, $\varphi_{1} \in\left[\varphi_{1}^{0}, \varphi_{1}^{1}\right]$, $\phi \in\left[\phi^{0}, \phi^{1}\right]$ and $\varphi_{2} \in\left[\varphi_{2}^{0}, \varphi_{2}^{1}\right]$ are used to represent the orientation of a grain. These three regions are uniformly divided into $n_{1}, n_{2}$ and $n_{3}$ small regions, respectively. Then, there are a total of $n_{1} \times n_{2} \times n_{3}$ sets of combinations of three different kinds of small regions. All grains whose three Euler angles fall within a set of combination are considered to belong to a state $h$, therefore $h$ contains $n_{1} \times n_{2} \times n_{3}$ values. 
In the following sections, the final results are shown. A random microstructure was produced by the computer program from which the statistical parameters were calculated. The two modes of deformation considered first are uniaxial tension and compression. Then the case of plane strain compression (approximated by rolling) will be discussed. For each of these tests, the imposed macroscopic velocity gradient $\overline{\mathbf{L}}$ is given. The form of $\overline{\mathbf{L}}$ is described below for each of the three tests.

tension along the $x$-axis

$$
\overline{\mathbf{L}}=\left[\begin{array}{rrr}
1 & 0 & 0 \\
0 & -\frac{1}{2} & 0 \\
0 & 0 & -\frac{1}{2}
\end{array}\right] L_{0},
$$

compression along the $x$-axis

$$
\overline{\mathbf{L}}=\left[\begin{array}{rrr}
-1 & 0 & 0 \\
0 & \frac{1}{2} & 0 \\
0 & 0 & \frac{1}{2}
\end{array}\right] L_{0},
$$

rolling in the $x-z$ direction

$$
\overline{\mathbf{L}}=\left[\begin{array}{rrr}
-1 & 0 & 0 \\
0 & 0 & 0 \\
0 & 0 & 1
\end{array}\right] L_{0} \text {. }
$$

Here $L_{0}$ is a constant. The equivalent strain is calculated from

$$
\bar{E}_{\mathrm{eq}}=\int_{0}^{t} \bar{D}_{\mathrm{eq}}(t) \mathrm{d} t
$$

where $\bar{D}_{\text {eq }}$ is the equivalent macroscopic strain rate and $t$ is time variable.

The equivalent strain rate and the equivalent deviatoric Cauchy stress, $\bar{\sigma}_{\text {eq }}$ and $\bar{D}_{\text {eq }}$, are defined by

$$
\begin{aligned}
& \bar{\sigma}_{\mathrm{eq}}=\left(\frac{3}{2} \bar{S}_{i j} \bar{S}_{i j}\right)^{1 / 2}, \\
& \bar{D}_{\mathrm{eq}}=\left(\frac{2}{3} \bar{D}_{i j} \bar{D}_{i j}\right)^{1 / 2},
\end{aligned}
$$

where $\bar{D}_{i j}$ is the macroscopic strain-rate component and $\bar{S}_{i j}$ is the macroscopic deviatoric Cauchy stress component. The parameters in Eq. (6) used in the numerical calculation are as follows: rate sensitivity coefficient $n=19$, the initial normalized reference stress for each slip system $\tau_{0}=1.00$, reference $\dot{\gamma}_{0}=0.001 / \mathrm{s}$. Strain hardening is neglected. For fcc materials, slip is assumed to occur on 12 slip systems $\left\{\begin{array}{llll}1 & 1 & 1\end{array}\right\}\left\langle\begin{array}{llll}1 & 1 & 0\end{array}\right\rangle$.

The logarithm of macroscopic strain-rate $\bar{D}_{\text {eq }}$ vs. the ratio of macroscopic stress $\bar{\sigma}_{\text {eq }}$ and reference stress $\sigma_{0}$ are shown in Figs. 3 and 4. for the cases of uniaxial tension and compression. Here, texture evolution has been ignored and the initial microstructure is 


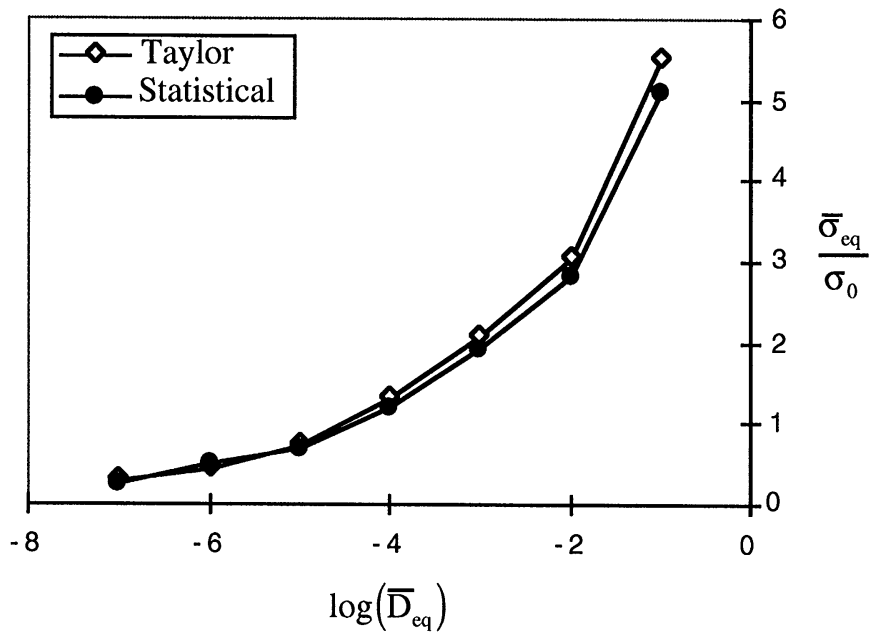

Fig. 3. Macroscopic behavior with different imposed macroscopic strain rate (uniaxial tension).

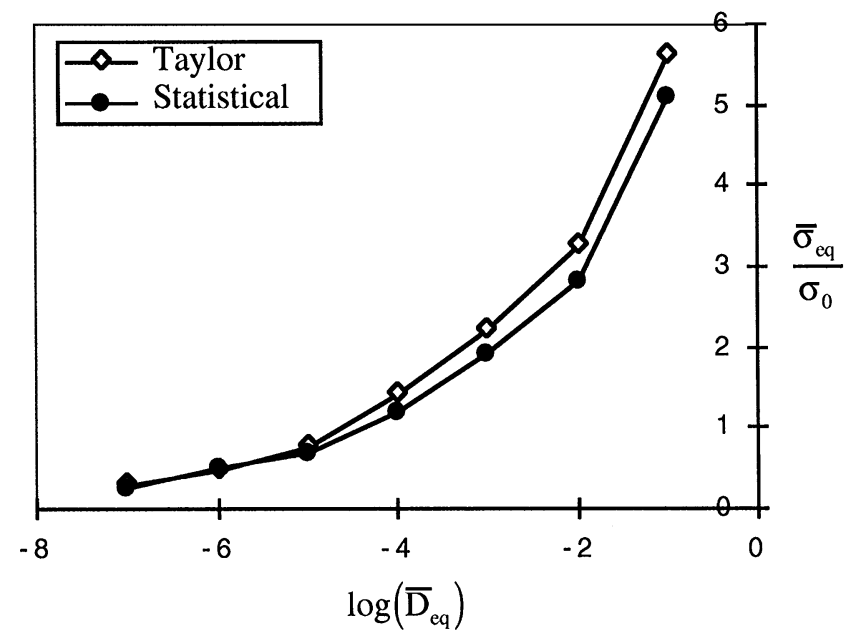

Fig. 4. Macroscopic behavior with different imposed macroscopic strain rate (uniaxial compression).

maintained during deformation. The prediction based on Taylor's model is also shown to be an upper bound compared to that of the statistical continuum results as expected because there are less constraints between grains in statistical model.

Figs. 5 and 6 describe the comparison of the macroscopic behaviors of the statistical model and Taylor's model for uniaxial tension and compression, respectively. The texture developments with macroscopic strain are shown by Figs. 7 and 8. During uniaxial tension, $\left\langle\begin{array}{llll}1 & 1 & 1\end{array}\right\rangle$ and $\left\langle\begin{array}{llll}1 & 0 & 0\end{array}\right\rangle$ fiber textures are formed, whereas the $\left\langle\begin{array}{llll}1 & 1 & 0\end{array}\right\rangle$ fiber texture is formed during uniaxial compression. 


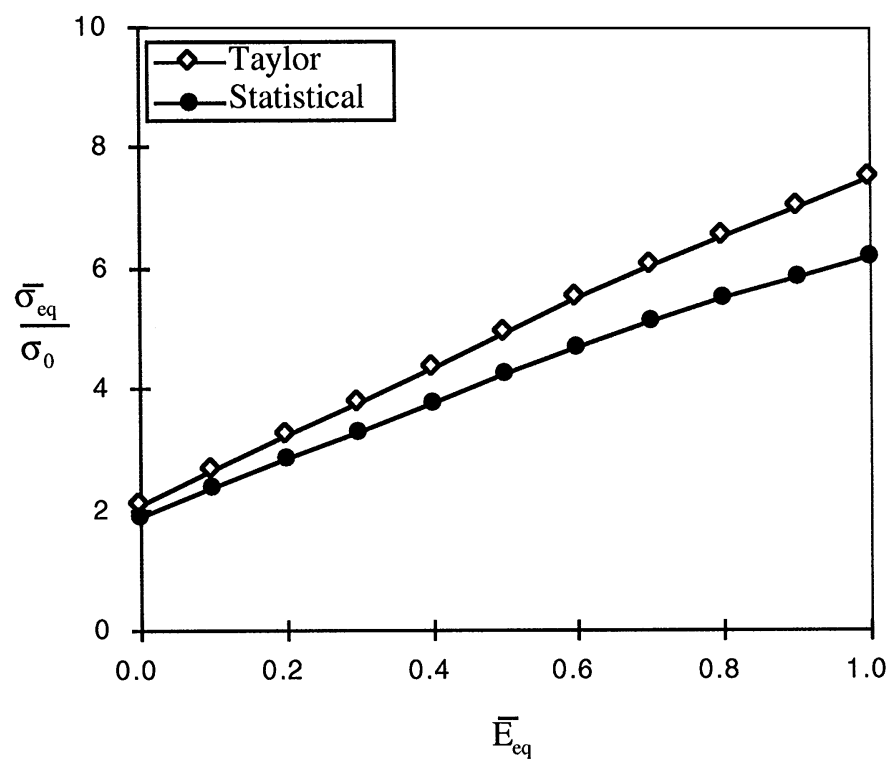

Fig. 5. Macroscopic behavior with macroscopic strain (uniaxial tension).

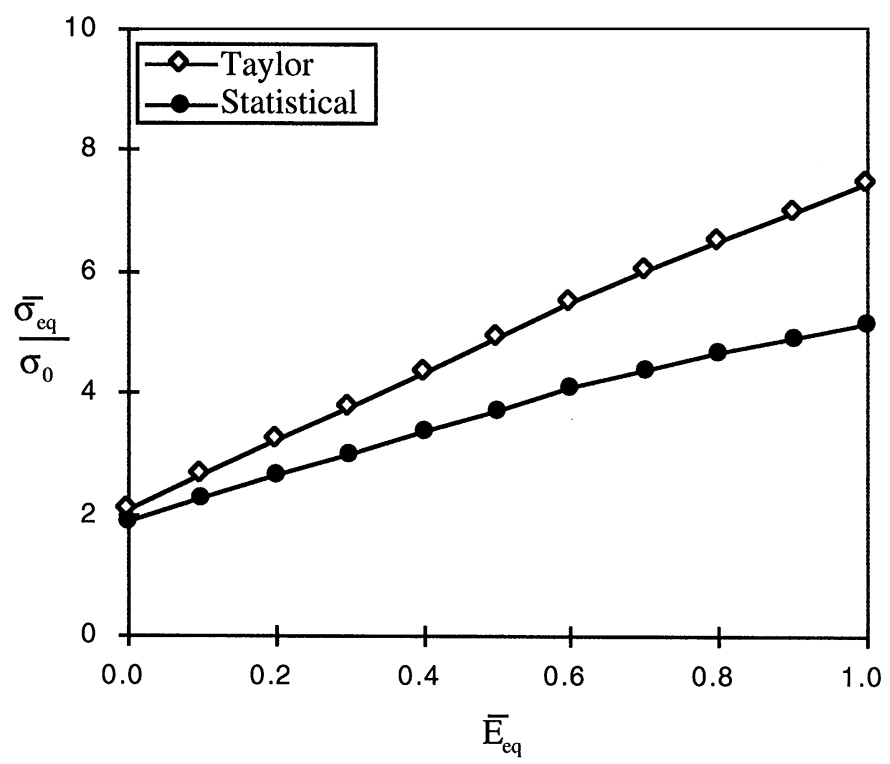

Fig. 6. Macroscopic behavior with macroscopic strain (uniaxial compression). 


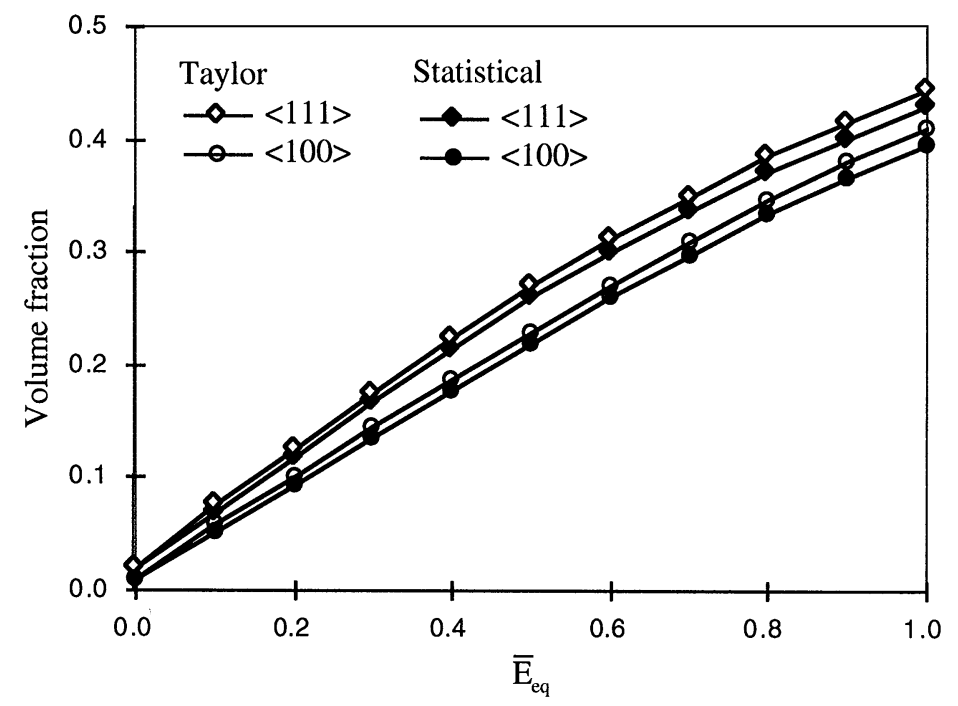

Fig. 7. The development of $\langle 111\rangle$ and $\langle 100\rangle$ texture with macroscopic strain (uniaxial tension).

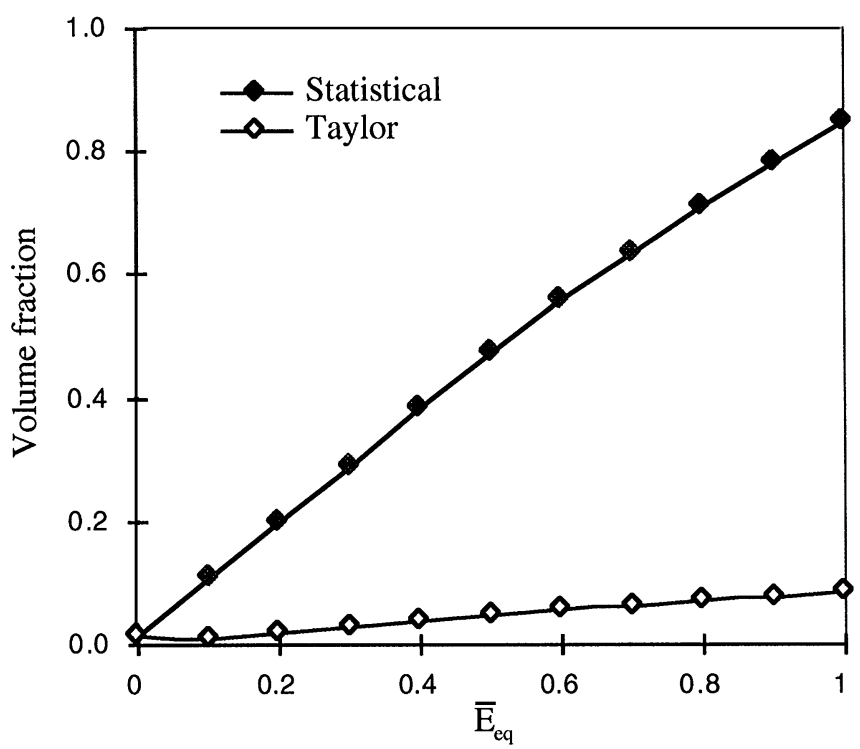

Fig. 8. The development of $\langle 110\rangle$ texture with macroscopic strain (uniaxial compression).

Fig. 9 shows the macroscopic behavior of the polycrystal under plane strain compression. Figs. 10 and 11 show the texture development as a function of the macroscopic strain. The simulation results are displayed in Fig. 10. There are three components

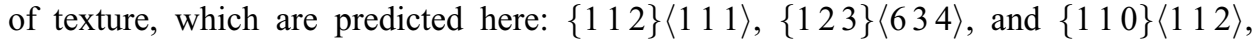
which are the copper, $\mathrm{S}$ component and brass types, respectively. 


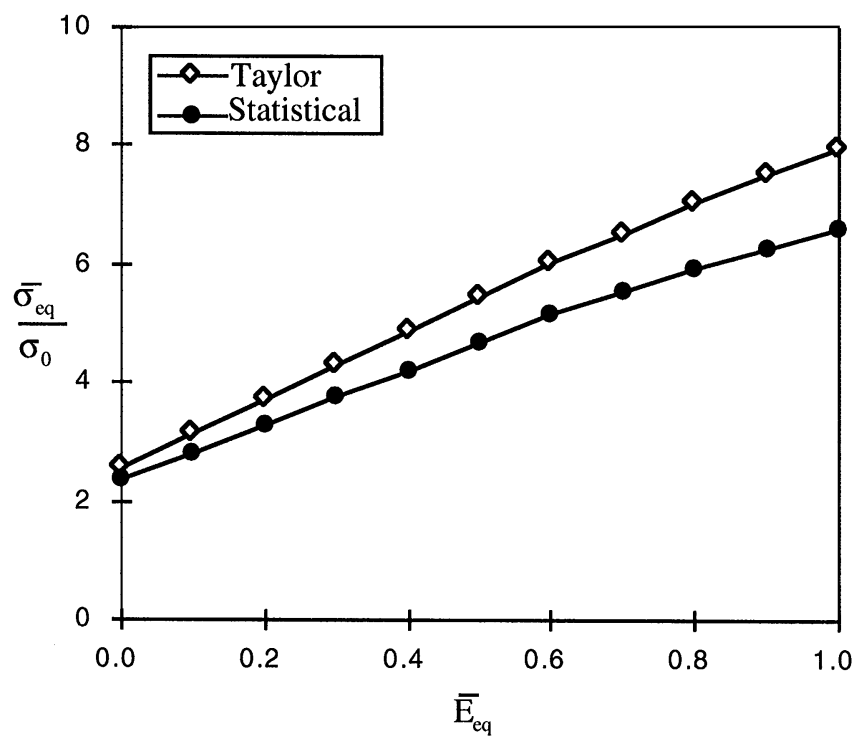

Fig. 9. Macroscopic behavior with macroscopic strain.

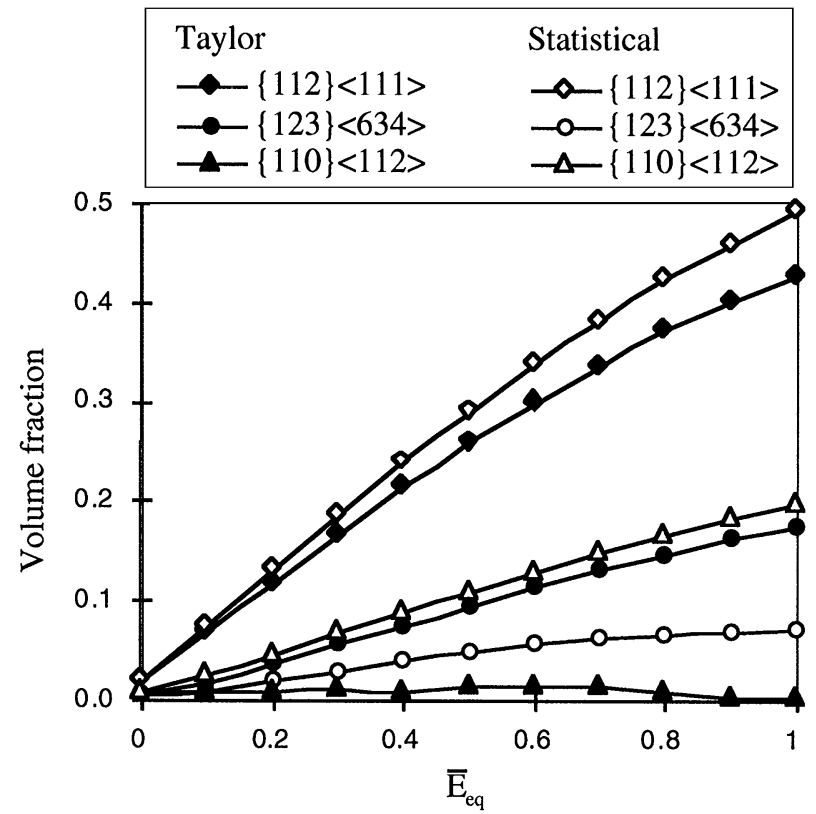

Fig. 10. The development of $\left\{\begin{array}{llllll}1 & 1 & 2\end{array}\right\}\left\langle\begin{array}{llll}1 & 1 & 1\end{array},\left\{\begin{array}{lll}1 & 1 & 0\end{array}\right\}\left\langle\begin{array}{lll}1 & 1 & 2\rangle\end{array}\right.\right.$ and $\left\{\begin{array}{llll}1 & 2 & 3\end{array}\right\}\langle 634\rangle$ texture with macroscopic strain (calculation results). 


\section{Discussion}

The results presented here show a clear evidence that the two-point correlations can rectify the weaknesses associated with the Taylor-type models in predicting texture components. In spite of the success which the theory of Taylor's (1938) has had, the inadequate predictions of the deformation textures in some cases show the inadequacy of this theory. The hypothesis of uniform strain implicitly assumes that the grains are of nearly equiaxed shape. Also the grain to grain contributions are entirely ignored. The results also show that for the case of tension and rolling there is very little contribution to the Taylor term. The only texture component, which seems to benefit the most from the two-point correlations are the brass component, which clearly does not evolve with deformation. The main success of the statistical simulation is clearly the axisymmetric compression. Taylor's model fails to correctly predict this component of texture.

The reason behind the failure of Taylor-type models in predicting the $\langle 110\rangle$ fiber texture for axisymmetric compression has been the topic of discussion for some time now and has been discussed in a recent book by Kocks et al. (1998). Barrett and Levenson (1940) have described the compression texture of aluminum as a very strong $\langle 110\rangle$ component with a tendency towards $\langle 312\rangle$. The results of Naaman et al. (1987) concerning the compression texture of copper show the strongest intensity to be near the $\langle 110\rangle$ component and in the band located between $\langle 110\rangle$ and $\langle 115\rangle$. When the grains are assumed as plates, Honneff and Mecking (1978) have observed that certain shear components may be present which are very different from those imposed on the matrix. Honneff and Mecking have modified the Taylor approach by imposing zero reaction stresses corresponding to these strain components. They have applied this approach to rolling and have obtained an improved prediction of textures for this mode of deformation. Kocks and Canova (1986) have generalized this approach for other cases, such as torsion and compression. This model is called the "RC" (relaxed constraint) or relaxed Taylor model. In support of the RC model, Tiem et al. (1986) have applied the concept to several cases showing that the RC model also simulates strong interactions among grains, but that certain strain components may be relaxed due to grain shape. The RC model however does not predict the experimental texture results in the case of compression, (Ahzi et al., 1990). This is due to the fact that the Taylor theory allows very little grain to grain interactions and the grains are not permitted to be deformed in the deformation plane. Asaro and Needleman (1985) have proposed an elasto-viscoplastic model for large deformations. This model is of Taylor type, since it assumes uniformity of the displacement gradient. It does not predict fiber $\langle 110\rangle$ texture either.

A self-consistent, large strain viscoplastic model has been proposed by Molinari et al. (1987). A remarkable difference between this model and Taylor-type models appears to be for the prediction of compression textures. The $\langle 110\rangle$ fiber is properly predicted for compression by the self-consistent scheme while the Taylor's does not predict this fiber. In the case of compression, the local strain rate of a significant percent of grains deviate from imposed macroscopic strain rate at a certain value. This effect, called the Hosford effect, Hosford (1964) results in a strong $\langle 110\rangle$ texture and 
is due to the fact that plain strain of neighboring grains is affected in different ways which accompany the slips to preserve the continuity of the material. Such an effect seems to be rectified once the grain to grain interactions are taken into account as is evident in self-consistent statistical mechanics model based on two-point correlation functions presented in this paper.

The two-point correlations functions allow the statistical mechanics model to take into account the grain shapes and the influence of individual grain on other neighboring grains. Detailed experimental and theoretical studies are under way to investigate such interactions. The major difference between the present model and the self-consistent models seems to be in the quantification of texture as shown in previous results. The present theory provides the volume fractions of grains with different orientations. Both ODF and MDF (misorientation distribution functions) can be predicted from these results. Self-consistent theories provide only general information of distribution of grains with different orientations, and therefore only pole figures can be obtained (Ahzi, 1987). No quantitative analysis can be performed by the present form of the self-consistent theories.

\section{Conclusions}

The statistical continuum theory has been applied to a polycrystalline material to predict the stress-strain response and texture evolution. Typical deformation textures have been successfully predicted by the model for FCC polycrystals. This includes the $\langle 111\rangle$ fiber under tension, the $\langle 011\rangle$ fiber under uniaxial compression and the well-known copper texture components during rolling (plane strain compression). The results for the uniaxial-axial compression overcome the well documented failures of the Taylor-like models to predict the $\langle 011\rangle$ fiber texture in FCC metals. The present theory emphasizes the secant-modulus form of single-crystal constitutive law originating with the work of Hutchinson. The secant modulus formulation is suited to the problem of creep, since the stresses typically remain a fraction of the yield stress. For moderate and large plastic deformation problems, the secant formulation may lead to stiff response. This is the case with the self-consistent models where it was shown that the secant formulation leads a response closer to the Taylor upper-bound predictions (see for instance the review by Molinari and Ahzi, 1997.) The tangent formulation of the self-consistent model, proposed by Molinari et al. (1987), gives softer response (lower flow stress). In the proposed statistical formulations, the predicted texture developments indicates that this formulation deviates appreciably from the Taylor upper bound. We also believe that if the tangent modulus formulation is used in the proposed framework, softer response will be predicted. The comparison of the two formulations (secant vs. tangent moduli) is underway and will be reported later. In comparison to the self-consistent theory, the major difference is in including the explicit distribution of microstructure surrounding each grain. The present theory provides a quantitative measure of texture based on the volume fractions of grains with different orientations from which ODF can be calculated. 


\section{References}

Adams, B.L., Canova, G.R., Molinari, A., 1989. A statistical formulation of viscoplastic behavior in heterogeneous polycrystals. Textures Microstruct. 11, 57-71.

Adams, B.L., Field, D.P., 1991. A statistical-theory of creep in polycrystalline materials. Acta Metall. Mater. 39 (10), 2405-2417.

Adams, B.L., Morris, P.R., Wang, T.T., Willden, K.S., Wright, S.I., 1987. Description of orientation coherence in polycrystalline materials. Acta Metall. 35, 2935-2946.

Adams, B.L., Wang, T.T., Morris, P.R., 1988. ICOTOM 8, Proceedings of the Eighth International Conference Textures of Materials, Santa Fe, p. 135.

Adams, B.L., Wright, S.I., Kunze, K., 1993. Orientation imaging: the emergence of a new microscopy. Metall. Trans. 24, 819.

Ahzi, S., 1987. A self-consistent approach for the viscoplastic behavior of polycrystals under large deformations. Ph.D. Thesis, Universite de Metz (France), France.

Ahzi, S., Molinari, A., Canova, G.R., 1990. Effect of the grain shape on the texture evolution of a polycrystalline material. In: Boehler, J.P. (Ed.), Yielding, Damage and Failure of Anisotropic Solids, EGF5. Mechanical Engineering Publ., London, pp. 425-441.

Asaro, R.J., Needleman, A., 1985. Texture development and strain-hardening in rate dependent polycrystals. Acta Metall. 33, 923-953.

Barrett, C.S., Levenson, L.H., 1940. Trans. AIME 137, 112.

Beran, M.J., Molyneux, J., 1966. Use of classical variational principles to determine bounds for the effective bulk modulus in heterogeneous media. Quart. Appl. Math. 24, 107.

Beran, M., Molyneux, J., 1968. Nuovo Cimento 30, 1406.

Bishop, J.F.W., Hill, R., 1951. A theoretical derivation of the plastic properties of a polycrystalline faced-centred metal. Philos. Mag. 42, 1298.

Budiansky, B., 1965. On the elastic moduli of some heterogeneous materials. Mech. Phys. Solids 13, 223-337.

Corson, P.B., 1976a. Correlation functions for predicting properties of heterogeneous materials. I. Experimental measurement of spatial correlation functions in multiphase solids. J. Appl. Phys. 45, 31593164.

Corson, P.B., 1976b. Correlation functions for predicting properties of heterogeneous materials. II. Empirical construction of spatial correlation functions for two phase solids. J. Appl. Phys. 45, 3165-3170.

Garmestani, H., Lin, S., 2000. Statistical continuum mechanics analysis of an elastic two-isotropic-phase composite material. J. Compos. Part B 31, 39-46.

Garmestani, H., Lin, S., Adams, B., 1998. Statistical continuum mechanics theory for inelastic deformation of a two phase medium. Int. J. Plasticity 14 (8), 719-731.

Harren, S.V., 1991. The finite deformation of rate dependent polycrystals-II: a comparison of the self-consistent and Taylor methods. J. Mech. Phys. Solids 39, 361-383.

Hashin, Z., Shtrikman, S., 1962. A variational approach to the theory of the elastic behaviour of polycrystals. J. Mech. Phys. Solids 10, 343-352.

Hill, R., 1965. Continuum micro-mechanics of elasticplastic polycrystals. J. Mech. Phys. Solids 13, 89-101.

Honneff, H., Mecking, H., 1978. Textures of Materials. Springer Berlin, p. 265.

Hosford, W., 1964. Trans-met Soc. AIME 230, 12.

Houtte, P. 1981. ICOTOM. vol. 6, p. 428.

Hutchinson, J.W., 1976. Bounds and self-consistent estimates for creep of polycrystalline materials. Proc. Roy. Soc. Lond. A 348, 101-127.

Klinskikh, S.D.V.a.N.A., 1965. Theory of the elastic properties of polycrystals. Fiz. Metal. Metalloved 19 (1), 25-32.

Kocks, U.F., Canova, G.R., 1981. Deformation of Polycrystals. Riso National Laboratory, Roskilde, Denmark.

Kocks, U.F., Canova, G.R., 1986. 34, Riso National Laboratory, Roshilde, Denmark.

Kocks, U.F., Tomé, C.N., Wenk, H.-R., 1998. Texture and Anisotropy. Cambridge University Press, Cambridge.

Kröner, E., 1967. Elastic moduli of perfectly disordered composite materials. J. Mech. Phys. Solids 15, 319-329. 
Kröner, E., 1972. Statistical Continuum Mechanics. Statistical Continuum Mechanics, Wien.

Kröner, E., 1987. In modelling small deformation of polycrystals. In: Zarka, e.J.G.a.J. (Ed.), Modelling Small Deformation of Polycrystals. Elsevier Applied Science Publishers, New York, pp. 229.

Lee, B.J., Ahzi, S., Asaro, R.J., 1995. On the plasticity of low symmetry crystals lacking five independent slip systems. Mech. Mater 20, 108

Leffers, T., 1968. Computer simulation of the plastic deformation in FCC polycrystals and the rolling texture. ICOTOM, vol. 1, pp. 1-33.

Lin, S., Garmestani, H., Adams, B.L., 2000. The evolution of probability functions in an inelasticity deforming two-phase medium. Int. J. Solids Struct. 37 (3), 423.

Lomakin, V.A., 1970. Statistical Problems of the Mechanics of Deformable Solids. Acad. Sciences, Moscow.

McCoy, J.J., 1981. Nemat-Naser, S. (Ed.), Macroscopic Response of Continua with Random Microstructures. Mechanics Today. Pergamon Press, New York, pp. 1-40.

Molinari, A., Canova, G.R., Ahzi, S., 1987. A self consistent approach of the large deformation polycrystal viscoplasticity. Acta Metall. 35, 2983-2994.

Morris, P.R., 1970. Elastic constants of polycrystals. Int. J. Eng. Sci. 8, 49-61.

Morris, P.R., Wang, T.T., Adams, B.L., 1988. ICOTOM 8, Proceedings of the Eighth International Conference on Textures of Materials, Santa Fe, p. 157.

Naaman, H., Talreja, R., Jensen, D.J., Hansen, N., 1987. Development of deformation textures in polycrystalline copper experiments and model predictions. Textures Microstruct. 7 (2), 149-170.

Nemat-Nasser, S., Obata, M., 1986. Rate-dependent, finite elastoplastic deformation of polycrystals. Proc. Roy. Soc. Lond. A407, 343-375.

Reuss, A., 1929. Berechnung der Fliessgrenze von Mischkristallen auf Grund der Plastizitatsbed-ingung fur Einkristalle. Z. Angew. Math. Mech. 9, 49-58.

Taylor, G.I., 1938. Plastic strain in metals. J. Inst. Metals 62, 307-325.

Tiem, S., Berveiller, M., Canova, G.R., 1986. Grain shape effects on the slip system activity and on the lattice rotations. Acta. Metall. 34 (11), 2139-2149.

Voigt, W., 1889. Uber die Beziehung zwischen den beiden Elastizitatskonstanten isotroper Korper. Wied. Ann. 38, 573-587.

Volkov, S.D., Klinskikh, N.A., 1965. Theory of the elastic properties of polycrystals. Phys. Metals Metallogr. $19,24$.

Wright, S.I., Adams, B.L., Kunze, K., 1993. Application of a new automatic lattice orientation measurement technique to polycrystalline aluminium. Mater. Sci. Eng. A 160, 229-240.

Zeller, R., Dederichs, P.H., 1973. Elastic constants of polycrystals. Phys. Stat. Sol. B 55(b), 831-841. 